\title{
Discoidin Domain Receptor 1 Is a Major Mediator of Inflammation and Fibrosis in Obstructive Nephropathy
}

\author{
Dominique Guerrot, ${ }^{\star \dagger \ddagger}$ Monique Kerroch, ${ }^{* \dagger}$ \\ Sandrine Placier, ${ }^{*}$ Sophie Vandermeersch, ${ }^{* \dagger}$ \\ Claire Trivin, ${ }^{*}$ Mouna Mael-Ainin, ${ }^{* \dagger}$ \\ Christos Chatziantoniou, ${ }^{* \dagger}$ and \\ Jean-Claude Dussaule ${ }^{\star \dagger}$ \\ From INSERM UMR S 702,* Hôpital Tenon, Paris; UPMC Paris 6 \\ University, ${ }^{\dagger}$ Paris; the Nephrology Department, ${ }^{\ddagger}$ Rouen University \\ Hospital, Rouen; and the Department of Physiology, ${ }^{\S}$ \\ Saint-Antoine Hospital, Paris, France
}

The interactions between tubulointerstitial infiltrating cells and the extracellular matrix play an important role in regulating renal fibrosis. Discoidin domain receptor 1 (DDR1) is a nonintegrin tyrosine kinase receptor for collagen implicated in cell adhesion, proliferation, and extracellular matrix remodeling. We have previously demonstrated that transgenic mice lacking DDR1 are protected from hypertension-associated renal fibrosis. The purpose of this study was to determine the role of DDR1 in renal inflammation and fibrosis related to primitive tubulointerstitial injury. After 12 days of unilateral ureteral obstruction (UUO), kidney histopathologic and real-time quantitative PCR analyses were performed in DDR1 ${ }^{-/-}$and wild-type mice. DDR1 expression was strongly increased in the obstructed kidney. Wild-type mice developed important perivascular and interstitial inflammation and fibrosis. In comparison, DDR1 ${ }^{-/-}$mice displayed reduced accumulation of fibrillar collagen and transforming growth factor $\beta$ expression. $\mathrm{F} 4 / \mathbf{8 0}^{+}$cell count and proinflammatory cytokines were remarkably blunted in $\mathrm{DDR}^{-/-}$obstructed kidneys. Leukocyte rolling and adhesion evaluated by intravital microscopy were not different between DDR1 $^{-/-}$and wild-type mice. Importantly, macrophages isolated from DDR1 ${ }^{-/-}$mice presented similar M1/M2 polarization but displayed impaired migration in response to monocyte chemoattractant protein-1. Together, these data suggest that DDR1 plays an important role in the pathogenesis of renal disease via enhanced inflammation. Inhibition of DDR1 expression or activity may represent a novel therapeutic target against the progression of renal diseases. (Am J Pathol 2011, 179:83-91; DOI: 10.1016/j.ajpath.2011.03.023)
Renal fibrosis is the consequence of the accumulation of extracellular matrix (ECM) components, including collagen, in the kidney. In chronic kidney diseases, irrespective of the initiating cause, fibrotic lesions autoaggravate, leading to a progressive decrease in renal function. Despite growing interest, the pathophysiologic pathways responsible for the progression of renal fibrosis remain elusive. A better understanding of specific mechanisms that promote inflammation and ECM synthesis is of critical importance in this setting because such pathways are susceptible to being at the cornerstone of the initiation and the progression of fibrogenesis.

Discoidin domain receptor 1 (DDR1) is a tyrosine kinase transmembrane receptor for collagen constitutively expressed in several cell types and organs, including the gastrointestinal tract, lung, and kidney. ${ }^{1}$ In the mammalian kidney, DDR1 is predominantly expressed by vascular smooth muscle cells, mesangial cells, and epithelial cells in normal conditions. ${ }^{2,3}$ On activation by binding to collagen I to VI and VIII, DDR1 regulates cell differentiation, adhesion, proliferation, and ECM remodeling. ${ }^{4-6} \mathrm{~A}$ number of studies have shown that DDR1 is implicated in carcinogenesis, inflammation, atherosclerosis, and fibrogenesis. ${ }^{7-12}$ Consistent with an important pathogenetic role in vascular diseases, ${ }^{8,13-15}$ we have demonstrated that DDR1-deficient mice were protected against renal vascular lesions induced by a long-term infusion of angiotensin II, a model in which hemodynamic alterations and vascular remodeling play a major role. ${ }^{11}$ Whether DDR1 may contribute to the initiating mechanisms and the progression of renal fibrosis irrespective of the initial cause remains unclear. Specifically, the implication of DDR1 remains to be established in lesions triggered by tubular injury. In addition, although exaggerated inflammation is identified as a major contributor to renal injury in a broad variety of diseases, the role of DDR1 has not yet

Supported by INSERM, UPMC Paris 6 University, and Rouen University Hospital.

Accepted for publication March 17, 2011.

Supplemental material for this article can be found at http://ajp. amjpathol.org or at doi: 10.1016/j.ajpath.2011.03.023.

Address reprint requests to Professor Jean-Claude Dussaule, M.D., Ph.D., INSERM UMR S 702, Hôpital Tenon, 75020 Paris, France. E-mail: jean-claude.dussaule@sat.ap-hop-paris.fr. 
been studied in a model that critically depends on renal inflammation. On this basis, the objective of the present study was to analyze the role of DDR1 in a tubulointerstitial model of inflammatory kidney disease induced by unilateral ureteral obstruction (UUO).

\section{Materials and Methods}

\section{Transgenic Mice}

Male transgenic mice aged 4 to 6 months and weighing 25 to $30 \mathrm{~g}$ were used in these experiments. The generation and genotyping of mice were described previously. ${ }^{13,16}$ The original background of the DDR1-null mice was a mix of $129 / \mathrm{Sv}$ with CD1. These mice have been backcrossed seven times to 129/Sv. No difference of the genetic background was found between DDR $1^{-/-}$ and wild-type (WT) controls after microsatellite analysis of DNA samples from 26 mice (13 DDR1 ${ }^{-1-}$ and $13 \mathrm{WT}$ ). The breeding couples that were used in our protocol were heterozygotes, and experiments were performed using DDR1-null mice and WT littermates. The mice were placed on regular diets and allowed free access to water. Overall, 55 mice were used for the present study. All animal procedures were in accordance with the European Union Guidelines for the Care and Use of Laboratory Animals.

\section{Unilateral Ureteral Obstruction}

After induction of general anesthesia (intraperitoneal injection of $50 \mathrm{mg} / \mathrm{kg}$ of pentobarbital), 12 DDR1-null mice and WT counterparts were subjected to a left flank incision. UUO was performed by complete ligation of the left ureter at the ureteropelvic junction, using double silk sutures. Nonobstructed sham kidneys were used as controls. Animals were sacrificed at day 12.

\section{Thioglycolate Peritonitis}

Thioglycolate peritonitis was induced in 11 WT and 9 DDR1-null mice with the intraperitoneal injection of $1 \mathrm{~mL}$ of sterile $4 \%$ thioglycolate solution (Sigma-Aldrich, St. Louis, MO). The mice were euthanized by decerebration 48 hours after injection, and the peritoneal cavities were washed with $5 \mathrm{~mL}$ of RPMI 1640 medium containing 20 mmol/L HEPES buffer and $0.1 \%$ bovine serum albumin. Cell counts were determined on Kova hemocytometer (Hycor Biomedical, Garden Grove, CA). Cells $\left(1 \times 10^{5}\right)$ were attached to glass slides with a Cytospin4 instrument (Thermo Fisher Scientific, Waltham, MA). Differential leukocyte counts were determined after May-Grünwald-Giemsa staining (VWR Scientific Products, Radnor, PA). The peritoneal lavage was incubated 2 hours at $37^{\circ} \mathrm{C}$ in $5 \% \mathrm{CO}_{2}$. Adhering macrophages $\left(2.5 \times 10^{5}\right)$ were placed in the upper chambers of Transwell $8-\mu \mathrm{m}$ pore size chemotaxis supports (Corning Inc, Corning, NY). Variable concentrations of mouse recombinant monocyte chemoattractant protein-1 (MCP-1) (R\&D Systems, Minneapolis, MN) were added in the lower chambers. After 3 hours, macrophages were counted with a Kova hemocytometer and the migration index was calculated as the percentage of macrophages having migrated to the lower chamber.

\section{Renal Histologic Analysis}

Cortical sections of obstructed and contralateral kidneys were immersed 24 hours in $4 \%$ formalin and thereafter embedded in paraffin after conventional processing (alcohol dehydration). Sections (3- $\mu$ m thick) were stained with Masson trichromic solution. The slides were independently examined on a masked basis for the level of perivascular and interstitial mononuclear cell infiltration, using a 0- to 4-point injury scale as described previously. ${ }^{17,18}$ Lesion indexes from individual sections were averaged to calculate a global index for each kidney.

\section{Sirius Red Morphometric Analysis}

Interstitial fibrosis was assessed on 8- $\mu$ m-thick, Sirius redstained paraffin sections at $\times 40$ magnification, under polarized light. Interstitial fibrosis was quantified using computerbased morphometric analysis software (Axionplan; Axiophot2, Zeiss, Germany). A specific stain pattern was selected and the percentage of the positive area in the examined tubulointerstitium was measured. Twelve cortical fields, excluding interlobular arteries, were selected randomly from each kidney. Scoring was performed in a masked manner on coded slides. Data are expressed as the mean value of the percentage of the positive area examined.

\section{Real-Time Quantitative PCR}

For the renal RNA extraction, both the obstructed and contralateral kidneys were harvested. RNA was extracted from renal cortex using TRIzol solution (Life Technologies BRL, Gaithersburg, MD). RNA was extracted from isolated peritoneal macrophages using RLT buffer lysis and purification with a Qiagen microkit. RNA quality was checked by measuring the ratio of optical densities at 260 and $280 \mathrm{~nm}$, and residual genomic DNA was removed by DNase I treatment for 30 minutes at $37^{\circ} \mathrm{C}$ (Fermentas Thermo Electron, Villebon sur Yvette, France). We used reverse transcription with a Revert Aid $\mathrm{H}$ minus First Strand DNA Synthesis kit (Fermentas) to convert $1 \mu \mathrm{g}$ of RNA into cDNA. cDNA was amplified by PCR with a LightCycler 480 (Roche Diagnostics, Meylan, France) using SYBR Green (Fast Start DNA Master SYBRGreen I; Roche Applied Science, Roche Diagnostics); specific primers for DDR1, MCP-1/CCL2, chemokine receptor 2 (CCR2), interferon- $\gamma($ IFN- $\gamma)$, transforming growth factor $\beta$ (TGF- $\beta$ ), collagen $3 \alpha_{1}$ chain (Col3A1), interleukin 23 (IL-23), tumor necrosis factor $\alpha$ (TNF- $\alpha$ ), macrophage mannose receptor 1 (MRC1), inducible nitric oxide synthase (iNOS), arginase 1 (Arg1), and glyceraldehyde-3-phosphate dehydrogenase (GAPDH) (for renal cortex); or the mean value of $\beta$ glucuronidase and hypoxanthine-guanine phosphoribosyltransferase (for peritoneal macrophages) as housekeeping genes under the following conditions: $95^{\circ} \mathrm{C}$ for 5 minutes, and 45 cycles at $95^{\circ} \mathrm{C}$ for 15 seconds and 
Table 1. Primers Used for Real-Time qPCR

\begin{tabular}{|c|c|c|}
\hline mRNA & Strand & Sequence \\
\hline DDR1 & $\begin{array}{l}\text { Sense } \\
\text { Antisense }\end{array}$ & 5'-CTCCACCCCATTCTGCAC-3' \\
\hline GAPDH & Sense & $\begin{array}{l}\text { 5 -CAGAAGGAGGCGG'AGGC-3 } \\
\text { 5'-AGCTTGTCATCAACGGGAAG-3' }\end{array}$ \\
\hline & Antisense & 5'-TTTGATGTTAGTGGGGTCTCG-3' \\
\hline $\mathrm{IFN}-\gamma$ & $\begin{array}{l}\text { Sense } \\
\text { Antisense }\end{array}$ & $\begin{array}{l}\text { 5'-ATCTGGAGGAACTGGCAAAA-3' } \\
5^{\prime}-\text { TTCAAGACTTCAAAGAGTCTGAGG-3' }\end{array}$ \\
\hline MCP1/ & Sense & 5' - GTTGGCTCAGCCAGATGCA-3' \\
\hline $\begin{array}{r}\text { CCL2 } \\
\text { TGF- } \beta 1\end{array}$ & $\begin{array}{l}\text { Antisense } \\
\text { Sense } \\
\text { Antisense }\end{array}$ & $\begin{array}{l}\text { 5'-AGCCTACTCATTGGGATCATCTTG-3' } \\
5^{\prime} \text {-TGGAGCAACATGTGGAACTC-3' } \\
5^{\prime}-\text { A }\end{array}$ \\
\hline IL-23 & $\begin{array}{l}\text { Antisense } \\
\text { Sense } \\
\text { Antisense }\end{array}$ & $\begin{array}{l}\text { 5'-GTCAGCAGCCGGTTACCA-3' } \\
5^{\prime}-\text { TCCCTACTAGGACTCAGCCAAC-3' } \\
5^{\prime} \text {-TGGGCATCTGTTGGGTCT-3' }\end{array}$ \\
\hline TNF- $\alpha$ & $\begin{array}{l}\text { Sense } \\
\text { Antisense }\end{array}$ & $\begin{array}{l}5^{\prime}-\text { TCTTCTCATTCCTGCTTGTGG-3' } \\
5^{\prime} \text {-ATGAGAGGGAGGCCATTTG-3' }\end{array}$ \\
\hline CCR2 & $\begin{array}{l}\text { Sense } \\
\text { Antisense }\end{array}$ & $\begin{array}{l}\text { 5'-ACCTGTAAATGCCATGCAAGT-3' } \\
5^{\prime}-\text { TGTCTTCCATTTCCTTTGATTTG-3' }\end{array}$ \\
\hline Gus B & $\begin{array}{l}\text { Sense } \\
\text { Antisense }\end{array}$ & $\begin{array}{l}5^{\prime} \text {-CTCTGGTGGCCTTACCTGAT-3' } \\
5^{\prime} \text {-CTCAGTTGTTGTCACCTTCACC-3' }\end{array}$ \\
\hline HPRT & $\begin{array}{l}\text { Sense } \\
\text { Antisense }\end{array}$ & $\begin{array}{l}5^{\prime} \text {-GGAGCGGTAGCACCTCCT-3' } \\
5^{\prime} \text {-CTGGTTCATCATCGCTAATCAC-3' }\end{array}$ \\
\hline Col3A1 & $\begin{array}{l}\text { Sense } \\
\text { Antisense }\end{array}$ & $\begin{array}{l}\text { 5'-TCCCCTGGAATCTGTGAATC-3' } \\
5^{\prime}-\text { TGAGTCGAATTGGGGAGAAT-3' }\end{array}$ \\
\hline iNOS & $\begin{array}{l}\text { Sense } \\
\text { Antisense }\end{array}$ & $\begin{array}{l}5^{\prime}-\text { GGGCTGTCACGGAGATCA-3' } \\
5^{\prime}-\text {-CCATGATGGTCACATTCTGC-3' }\end{array}$ \\
\hline MRC1 & $\begin{array}{l}\text { Sense } \\
\text { Antisense }\end{array}$ & $\begin{array}{l}5^{\prime}-\text { CCACAGCATTGAGGAGTTTG-3' } \\
5^{\prime}-\text { ACAGCTCATCATTTGGCTCA-3' }\end{array}$ \\
\hline Arg1 & $\begin{array}{l}\text { Sense } \\
\text { Antisense }\end{array}$ & $\begin{array}{l}5^{\prime} \text {-CCTGAAGGAACTGAAAGGAAAG-3' } \\
5^{\prime}-\text { TTGGCAGATATGCAGGGAGT-3' }\end{array}$ \\
\hline
\end{tabular}

$60^{\circ} \mathrm{C}$ for 15 seconds, then $72^{\circ} \mathrm{C}$ for 15 seconds. Specific primers were designed by Universal Probe Library system (Roche Applied Science) (primers are detailed in Table 1). To normalize the quantitative PCR (qPCR) results, we used Roche LightCycler 2.0 software (Roche Diagnostics). We expressed results as $2^{-} \Delta \mathrm{Cp}$, where $\mathrm{Cp}$ is the cycle threshold number. We analyzed dissociation curves after each run for each amplicon to assess the specificity of the quantification when using SYBR Green.

\section{IHC for DDR1, Megalin, CD3, F4-80, Mac1, and Dendritic Cells}

Cryostat sections (4- $\mu \mathrm{m}$ thick) of renal cortex were fixed with acetone for 7 minutes. After blockade of endogenous peroxidase, they were stained with an anti-DDR1 $(4 \mu \mathrm{g} / \mathrm{mL}$; C-20, Santa Cruz Biotechnology, Santa Cruz, CA), antiCD3 $\varepsilon$ (Santa Cruz), anti-F4-80 (AbCys, Paris, France), and antimegalin (Santa Cruz), and the Envision kit (Dako, Carpinteria, CA) was applied for 30 minutes at room temperature. Staining was revealed by applying the DAB kit (Dako), hematoxylin QS (Vector, Burlingame, CA), and Permanent Mounting Media (Aqueous) (Innovex, Richmond, VA). Additional experiments with DDR1-specific blocking peptide were performed (Santa Cruz). For semiquantitative analysis of DDR1 expression in vessels, glomeruli, interstitium, and tubular epithelium, slides were independently examined on a masked basis, using a 0- to 4-point relative intensity scale. Indexes from individual sections were averaged to calculate a global index for each kidney. The double-stain experiments were performed using frozen sec- tions fixed to acetone for 7 minutes and then washed with PBS using anti-DDR1, anti-Mac1 (BD Pharmingen, Le Pont de Claix, France), anti-dendritic cell (MCA948; AbD Serotec, Raleigh, NC), anti-rabbit IgG tetramethyl rhodamine isothiocyanate (DDR1) (Jackson Immunoresearch, West Grove, PA), anti-rabbit fluorescein isothiocyanate (DDR1), anti-rat tetramethyl rhodamine isothiocyanate (Mac1, MCA948), and rat anti-mouse F4/80 fluorescein isothiocyanate (AbD Serotec). Immunofluorescence micrographs were obtained using an Olympus BX 51 camera DP70 (Olympus, Rungis, France).

\section{Intravital Microscopy}

After induction of general anesthesia (intraperitoneal injection of $50 \mathrm{mg} / \mathrm{kg}$ of pentobarbital) and intraperitoneal injection of $1 \mu \mathrm{g} / \mathrm{kg}$ of mouse recombinant MCP-1 (R\&D), 10 DDR1-null mice and WT counterparts were maintained at $37^{\circ} \mathrm{C}$ on a heated microscope stage, using a rectal electrothermometer (CMA, Solna, Sweden). A PE10 catheter (Phymep, Paris, France) was placed in the right femoral vein for venous access and continuous $50-\mu \mathrm{L} / \mathrm{min}$ infusion of $2.5 \%$ bovine serum albumin in $0.9 \%$ saline. After midline abdominal incision, the mesentery adjoining the terminal ileum was carefully placed on a glass pedestal (Klaus Effenberger Medizinisch Technicher Gerätebau, Germany) and superfused with $0.9 \%$ saline. Intravenous injection of $250 \mu \mathrm{g}$ of rhodamine 6G (R4127; Sigma-Aldrich) was performed to label circulating leukocytes. Imaging was performed using a fixed-stage, upright microscope with a water immersion $\times 40$ objective (Axio, Examiner Z1; Zeiss). Single unbranched mesenteric veins ranging from 80 to 120 $\mu \mathrm{m}$ in diameter were studied, and the microcirculation was recorded during consecutive 20-second periods in at least 3 different mesenteric veins per animal (Axiovision 4 software; Zeiss). The mean duration of the experimental procedure, from injection of MCP-1 to sacrifice, did not exceed 90 minutes. Quantification of leukocyte adhesion and rolling were performed off-line during a single-session, masked playback analysis of videotaped images (ImageJ software). Leukocytes were defined as adherent when immobile during at least 10 seconds and were expressed as the number of cells adhering on a $100-\mu \mathrm{m}$ vein length per 20 -second period. Rolling leukocytes were defined as cells that were clearly moving along the endothelium slower than free-flowing blood cells and expressed as the number of rolling cells per millimeter and per second.

\section{Statistical Analyses}

Statistical analyses were performed using analysis of variance followed by Fisher's Protected Least Significance Difference test (Statview software version 5.0; SAS Institute, Cary, NC). Results with $P<0.05$ were considered statistically significant. All values are means \pm SEM. 

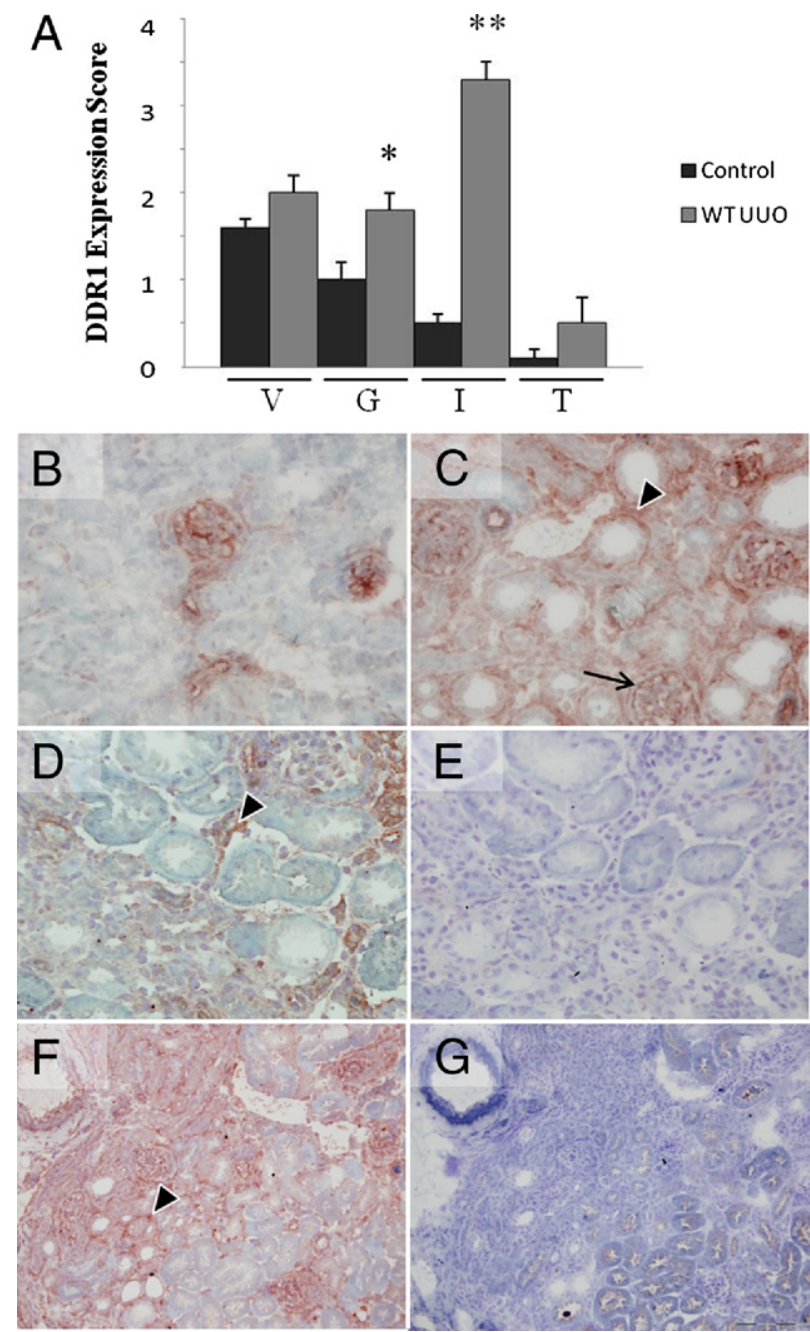

Figure 1. DDR1 expression is induced in the interstitium of the obstructed kidney. A: Semiquantitative evaluation of DDR1 expression in the kidney 12 days after UUO in vessels (V), glomeruli (G), interstitium (I), and tubular epithelium (T). B-G: DDR1 immunostaining in the control kidney. B: In control kidney, predominant expression is in the arteriolar wall and in the mesangium $(\times 40)$. Then 12 days after UUO, DDR1 is strongly induced in interstitial cells (arrowheads) $(\mathbf{C}$ and $\mathbf{D}, \times 40 ; \mathbf{F}, \times 20)$. Note a focal de novo expression on glomerular epithelial cells (arrow). E: Negative control $(\times 40)$. F and G: Proximal tubular epithelial cells, identified by megalin-positive apical staining, do not significantly coexpress DDR1, as evidenced by DDR1 $(\mathbf{F})$ and megalin $(\mathbf{G})$ stainings on consecutive sections $(\times 20)$. Data are mean \pm SEM. ${ }^{*} P<0.05,{ }^{* *} P<0.01$ versus nonobstructed WT kidney.

\section{Results}

\section{DDR1 Expression Is Induced in the Obstructed Kidney}

At baseline, DDR1 is expressed in the mouse kidney, as evidenced by real-time qPCR and immunohistochemistry (IHC). In control kidneys, DDR1 showed a predominant localization in the vascular wall and in the mesangium (Figure 1B). After 12 days of UUO, real-time qPCR demonstrated a 30-fold increase in DDR1 expression (DDR1/ GAPDH: $0.7 \pm 0.3 \mathrm{AU}$ in control kidneys versus $19.1 \pm 4.1$ in UUO; $P<0.01)$. DDR1 expression in the obstructed kidney was mainly increased in the interstitium and in glomeruli, as shown by the semiquantitative evaluation (Figure

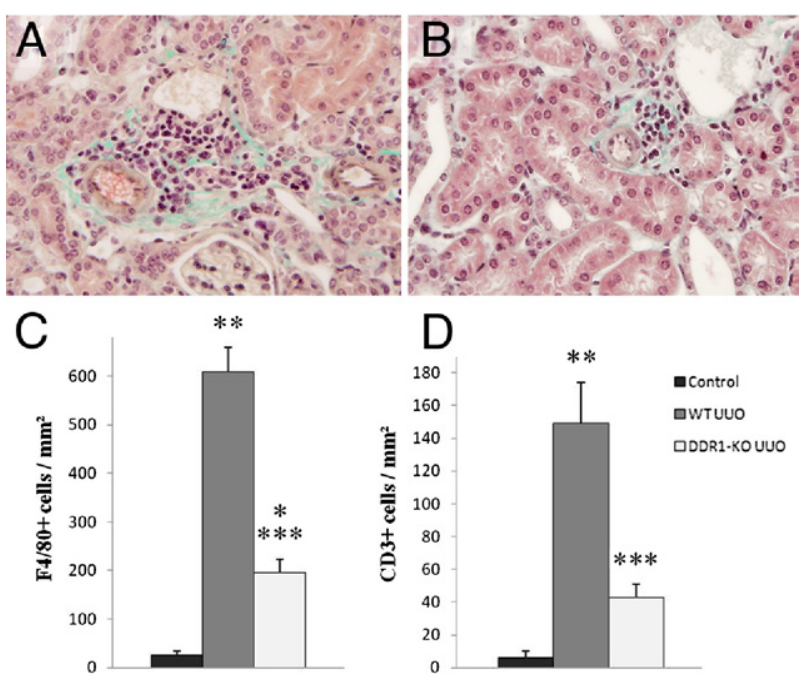

Figure 2. DDR $1^{-/-}$mice present reduced accumulation of inflammatory cells in the obstructed kidney. Representative views of Masson trichrome stainings in obstructed kidneys in WT $(\mathbf{A}, \times 40)$ and DDR1 $1^{-/-}$mice $(\mathbf{B}, \times 40)$. Note the cellular infiltration in the vicinity of the vessels. Cortical cell count for F $4 / 80^{+}$ cells $(\mathbf{C})$ and $\mathrm{CD}^{+}$cells $(\mathbf{D})$ in control, WT UUO, and DDR $1^{-/-}$UUO kidneys, expressed per unit area. Data are mean \pm SEM. ${ }^{*} P<0.05$, ${ }^{* *} P<0.01$ versus nonobstructed kidney; ${ }^{* * * *} P<0.01$ versus obstructed WT kidney.

1A). Immunohistochemistry revealed a striking de novo expression of DDR1 in interstitial cells and a focal expression on glomerular parietal epithelial cells (Figure 1, C, D, and F), whereas megalin-positive proximal tubular epithelial cells, which undergo major structural and functional changes in the UUO model, were not positively stained for DDR1 (Figure 1G).

\section{Renal Inflammation Is Reduced in DDR1-Null Mice}

We performed an analysis of the mononuclear cell infiltration on Masson trichrome staining to evaluate the in-
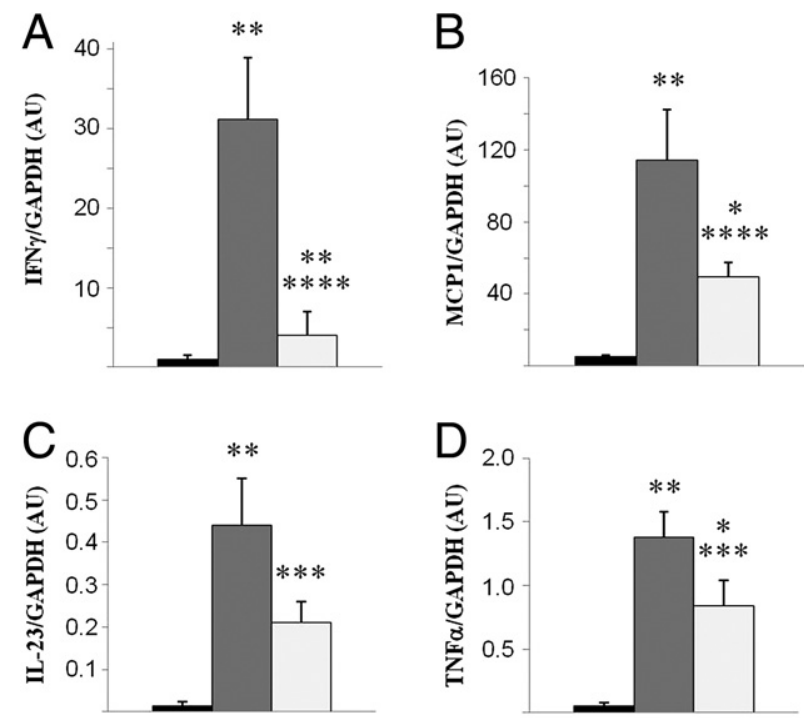

Figure 3. DDR $1^{-/-}$mice present reduced expression of proinflammatory cytokines in the obstructed kidney. Expression of IFN- $\gamma(\mathbf{A})$, MCP1/CCL2 (B), IL-23 (C), and TNF- $\alpha$ (D) evaluated by real-time qPCR. Control nonobstructed kidneys (black bars), WT UUO (gray bars), and DDR1-KO UUO (white bars). Data are mean \pm SEM. ${ }^{*} P<0.05,{ }^{* *} P<0.01$ versus nonobstructed kidney; ${ }^{* * * * *} P<0.05,{ }^{* * * * * *} P<0.01$ versus obstructed WT kidney. 

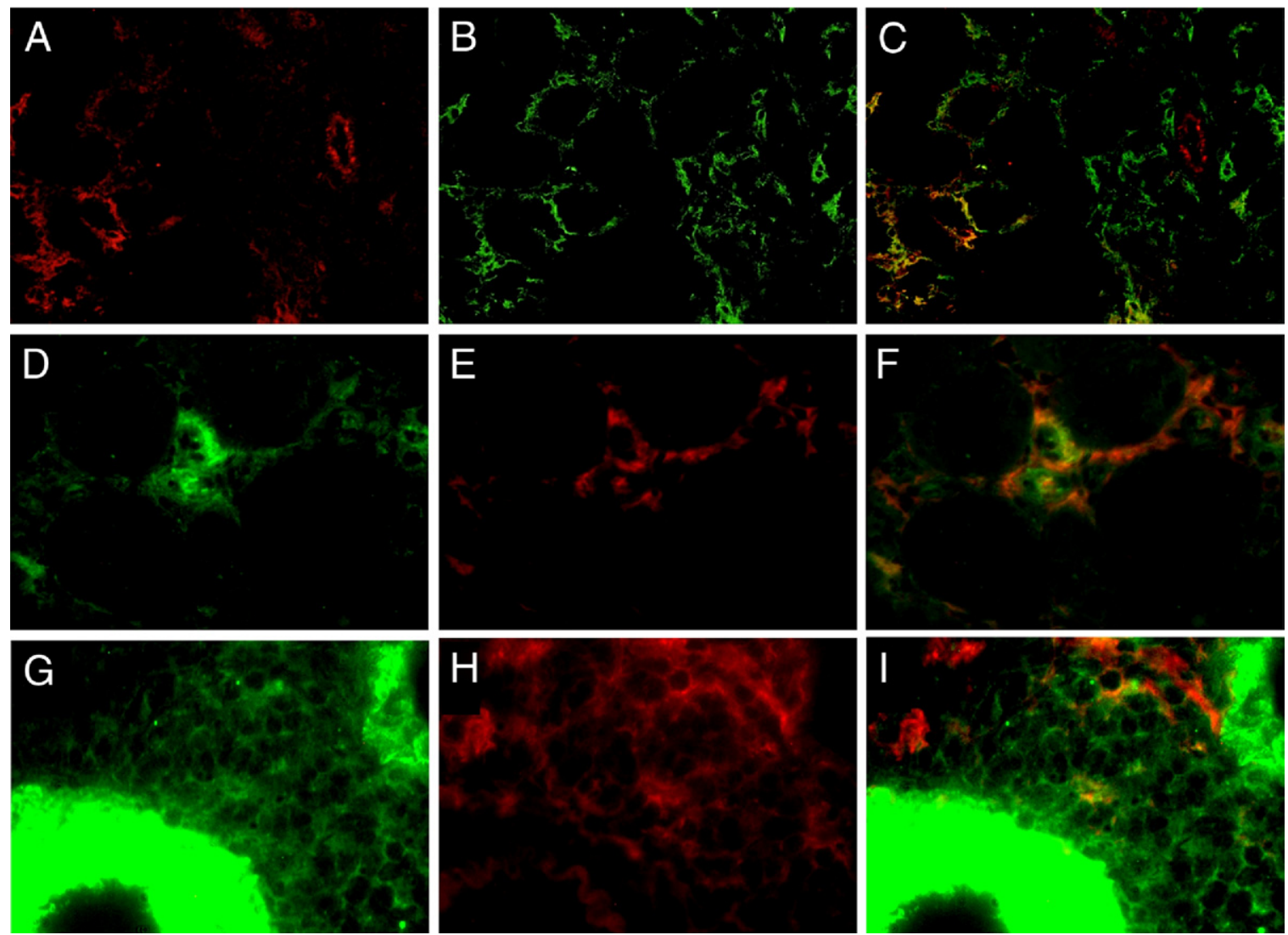

Figure 4. Double immunostainings for DDR1 (A, D and $\mathbf{G}$ ) with F4/80 (B, merge in $\mathbf{C})$, Mac1 (E, merge in $\mathbf{F}$ ), and dendritic cell marker MCA948 (H, merge in I) in renal cortex 12 days after UUO. Merge shows partial overlapping, consistent with the predominant expression of DDR1 by a subset of the macrophages recruited in the interstitium of the obstructed kidney $(\mathbf{A}-\mathbf{C}, \times 20 ; \mathbf{D}-\mathbf{I}, \times 40)$.

fluence of DDR1 deficiency on the development of postobstructive renal inflammation. As expected, in WT mice, UUO induced striking perivascular inflammation $(0.1 \pm$ 0.1 in control kidneys versus $2.6 \pm 0.3$ in UUO; $P<0.01$ ) (Figure 2A). The degree of perivascular inflammation was significantly blunted in DDR1-null mice $(1.9 \pm 0.2$ versus $2.6 \pm 0.3$ in WT UUO; $P<0.05$ ) (Figure 2B). Semiquantitative analysis of interstitial inflammation showed a similar protection $\left(1.7 \pm 0.2\right.$ in DDR1 $1^{-1-} \mathrm{UUO}$ versus $2.4 \pm 0.3$ in WT UUO; $P<0.05$ ). Quantitative analyses of specific immunostainings showed that $\mathrm{F} 4 / 80^{+}$cells and $\mathrm{CD}^{+}$lymphocytes were significantly reduced in DDR1 ${ }^{-1-}$ mice compared with WT mice (Figure 2, C and D). Expression of the proinflammatory cytokine IFN- $\gamma$, evaluated by real-time qPCR, was induced by UUO and was significantly blunted in DDR1 $1^{-1-}$ mice (Figure 3A). MCP-1, IL-23, and TNF- $\alpha$ were also diminished in DDR1-null mice compared with the WT mice (Figure 3, B-D).

\section{In UUO, a Subpopulation of the Interstitial Macrophages Expresses DDR1}

Although T lymphocytes were principally located in perivascular infiltrates in WT and DDR1 ${ }^{-1-}$ mice, $\mathrm{F} 4 / 80^{+}$ and $\mathrm{Mac} 1^{+}$cells showed a predominant interstitial distribution. Owing to the similar interstitial pattern of DDR1 de novo staining (Figure 1, C, D, and F), we addressed the question of a coexpression of DDR1 and F4/80 in infiltrating interstitial cells in the obstructed kidney. We therefore performed double-staining immunofluorescence for DDR1 and F4/80 in WT UUO kidneys. Fluorescence microscopy analysis revealed a partial coexpression of these two antigens in interstitial cells (Figure 4, A-C). Approximately $50 \%$ of the $\mathrm{F} 4 / 80^{+}$cells presented a coexpression of DDR1. Because F4/80 may be expressed by macrophages and dendritic cells in the obstructed kidney, we performed additional immunostainings to better characterize this population of interstitial cells. We found that DDR1 was predominantly colocalized with Mac1 (Figure 4, D-F) and to a lesser extent with the dendritic cell marker MCA948 (Figure 4, G-I).

\section{Macrophages Isolated From DDR1-Null Mice Present Reduced Migration}

Leukocyte invasion is critical for tissue inflammation. We have previously shown that DDR1-deficient mice and WT counterparts present no quantitative difference in circulating leukocytes, lymphocytes, monocytes, and neutrophils. ${ }^{11}$ Here, we have found that a subset of the interstitial leukocytes recruited after UUO expressed DDR1. Because DDR1-null mice presented reduced inflammation in the obstructed kidney, we next addressed the issue of a proinflammatory role of DDR 1 in macrophages 

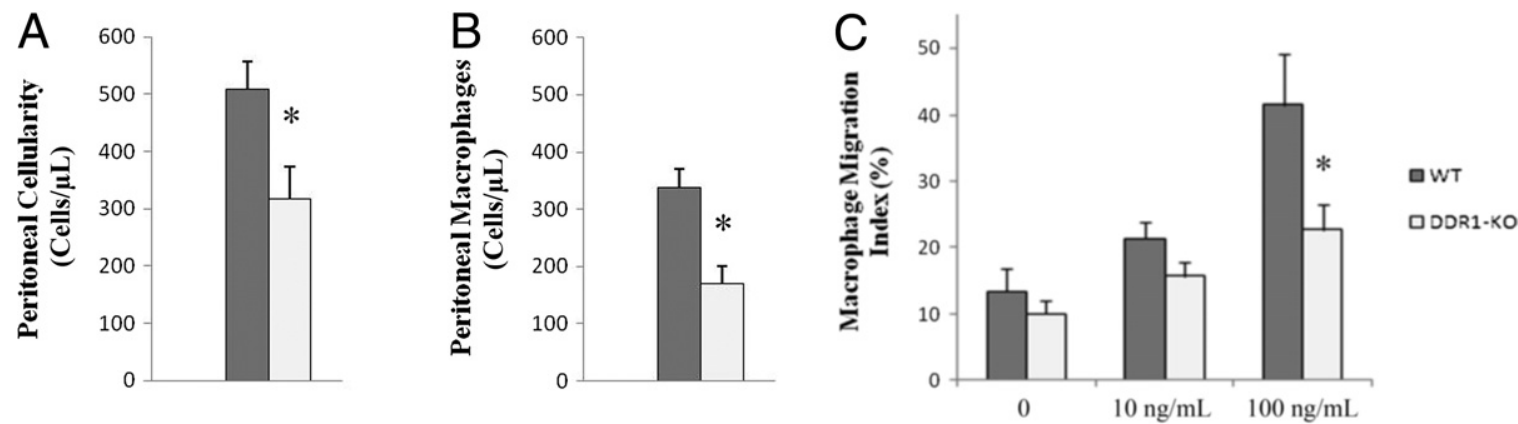

Figure 5. Macrophages isolated from DDR1 ${ }^{-/-}$mice present reduced migration ability. Global cellularity (A) and monocyte/macrophages cell count in peritoneal lavages (B). Macrophages migration assay in Boyden chambers in response to increasing concentrations of MCP-1 (C). Data are mean \pm SEM. ${ }^{*} P<0.05$ DDR $1^{-/-}$ versus WT evaluated independently by analysis of variance, followed by Fisher's Protected Least Significance Difference test.

via increased migration or differential M1/M2 polarization. To this end, we isolated macrophages from DDR $1^{-1-}$ and WT mice 48 hours after induction of an aseptic peritonitis by injection of thioglycolate. As illustrated in Figure 5, A and $\mathrm{B}$, the overall cellular count and the specific macrophage count in the peritoneal lavages were reduced in DDR1-null mice. Double immunostainings and real-time qPCR revealed that the peritoneal $\mathrm{F} / 80^{+}$cells expressed DDR1 (Figure 6, A-C). Macrophages isolated from the peritoneal cavity were allowed to migrate against increasing concentrations of MCP-1. Importantly, DDR1null macrophages exhibited a significantly reduced migration compared with cells isolated from WT mice (Figure $5 \mathrm{C}$ ). As shown in Figure 7A, macrophages isolated from DDR1 ${ }^{-1-}$ and WT mice presented no significant difference in the expression of CCR2, the macrophage receptor for MCP-1/CCL2, whereas CCR2 was reduced in the obstructed kidney of DDR1 ${ }^{-1-}$ mice, consistent with blunted inflammatory infiltrate (Figure 7B). The chemotactic signal-inducing macrophage migration in our in vitro conditions was consequently similar in both strains. Because macrophages may represent a heterogeneous population, we thereafter performed real-time qPCR analyses of selected M1- and M2-associated mRNA on isolated macrophages to determine whether DDR1 influenced M1/M2 phenotype. We found no significant difference between DDR $1^{-1-}$ and WT macrophages in all of the M1 (iNOS, MCP-1, and IL-23) and M2 (Arg1 and MRC1) markers analyzed in this study. TNF- $\alpha$ and TGF- $\beta$ mRNA was similar between DDR1 ${ }^{-1-}$ and WT macrophages. Col3A1 expression was low in macrophages and did not exhibit strain-dependent difference (see Supplemental Figure S1 at http://ajp.amjpathol.org).

\section{Rolling and Adhesion of Circulating Leukocytes Are Not Altered in DDR1-Null Mice}

To determine whether reduced leukocyte infiltration in DDR $1^{-/-}$mice may be due to impaired interactions between circulating leukocytes and the vascular endothelium, we evaluated the rolling and adhesion of MCP-1 chemoattracted leukocytes in vivo. The analyses of these intravital microscopy experiments showed no difference between DDR1 ${ }^{-1-}$ and control WT mice in leukocyte rolling and adhesion in mesenteric veins (Figure 8, A and B; see also compressed Supplemental Video S1 at http:// ajp.amjpathol.org).

\section{DDR1-Null Mice Are Protected Against Interstitial Fibrosis Induced by UUO}

We next investigated the effect of DDR1 deficiency on the development of renal interstitial fibrosis. Interstitial fibrillar collagen accumulation was studied by morphometric analysis in Sirius red-stained renal sections as an index of the fibrotic response to UUO. After 12 days of UUO, the Sirius red score showed a 12-fold increase in the obstructed kidney in WT mice compared with control kidneys. DDR $1^{-1-}$ mice presented a $33 \%$ reduction in the accumulation of interstitial collagen compared with the WT mice (Figure 9, A-C). The real-time qPCR evaluation of the UUO-induced expression of Col3A1 and TGF- $\beta 1$, a key profibrotic cytokine, was significantly lower in DDR $1^{-1-}$ than in WT mice, consistent with reduced fibrogenesis in DDR1 ${ }^{-1-}$ mice (Figure 9, D and E).
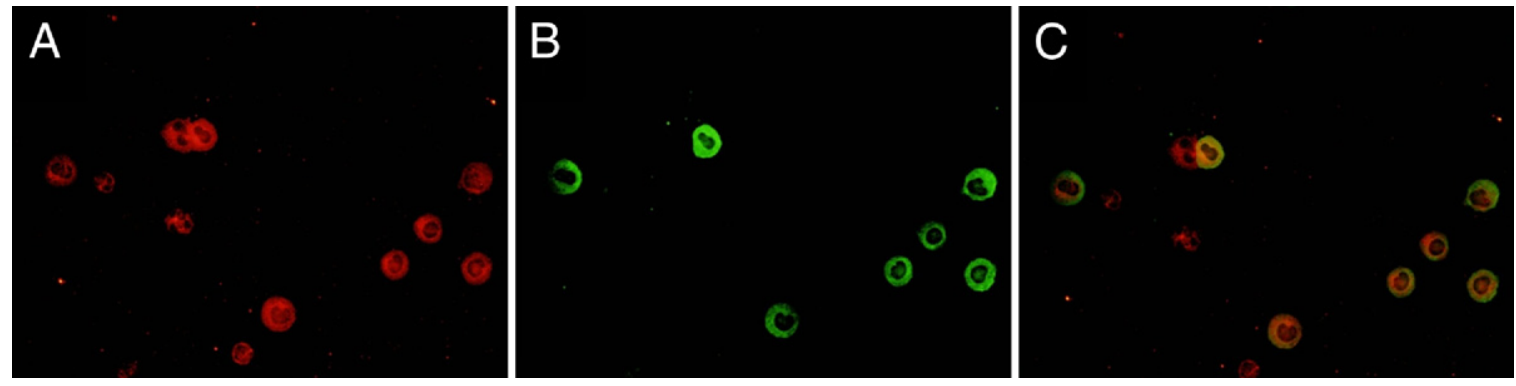

Figure 6. Double immunostainings for DDR1 $(\mathbf{A}, \times 60)$ and $\mathrm{F} 4 / 80(\mathbf{B}, \times 60)$ in peritoneal lavages, 48 hours after thioglycolate injection. Merge $(\mathbf{C}, \times 60)$ 

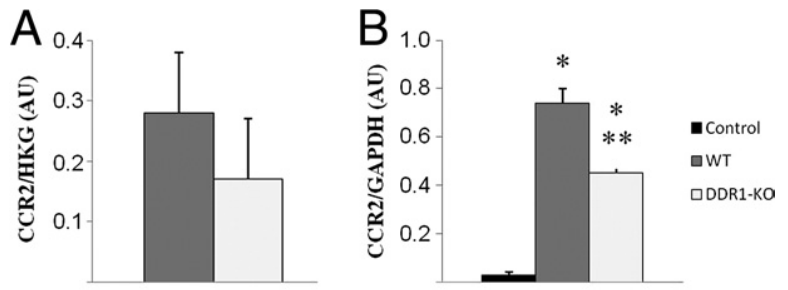

Figure 7. Expression of the MCP1/CCL2 ligand, CCR2, on isolated peritoneal macrophages $(\mathbf{A})$ and on the renal cortex $(\mathbf{B})$. Data are mean \pm SEM. ${ }^{*} P<$ 0.01 versus nonobstructed kidney; ${ }^{* * *} P<0.01$ versus obstructed WT kidney.

\section{Discussion}

Because it regulates interactions between the ECM and both resident and infiltrating cells, DDR1 is a potent actor of tissue adaptations to acute and chronic lesions. In this study, we show that DDR1 plays a crucial role in renal injury induced by UUO. We demonstrate that DDR1-deficient mice have reduced inflammation and subsequent accumulation of fibrillar collagen in the obstructed kidney and that interstitial infiltrating cells are important mediators of the expression of DDR1 in renal inflammation. We further identify a subpopulation of DDR1 ${ }^{+}$macrophages in the obstructed kidney and demonstrate impaired migration in DDR1 ${ }^{-1-}$ macrophages. These results focus attention on a direct role of DDR1 in the initiation and progression of renal inflammatory diseases.

In normal conditions, DDR1 is expressed in the kidney. ${ }^{1-3}$ However, current evidence does not support a key role for DDR1 in renal development and physiology, in particular because transgenic mice lacking DDR1 present a close to normal renal phenotype in basal conditions. ${ }^{11,19}$ In contrast, DDR1 has been established as an important contributor to a broad variety of pathologic processes, especially in cancer and inflammation, by promoting cell migration, invasion, and survival..$^{9,10,20-23}$ In parallel, several in vitro and in vivo studies have shown that DDR1 was involved in crucial mechanisms leading to fibrogenesis. ${ }^{6,12,24}$ However, to date few studies have analyzed the implications of DDR1 in the setting of kidney fibrosis.

We have previously demonstrated that DDR1-deficient mice presented a blunted degree of collagen I and IV expressions during hypertension-induced renal fibrosis. ${ }^{11}$ This study revealed reduced renal inflammation in the transgenic mice, which suggests indirect consequences of DDR1 activation on the progression of renal fibrosis through enhanced local inflammation. Such a
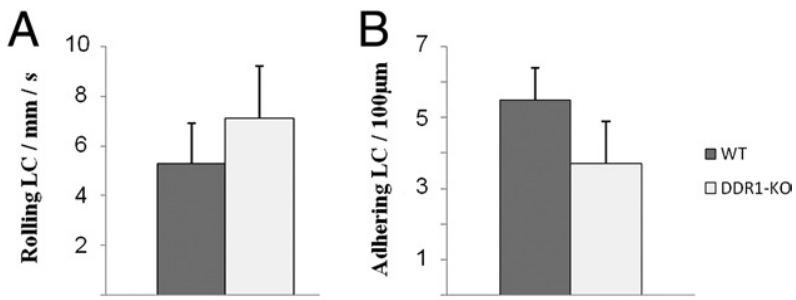

Figure 8. Quantification of leukocyte rolling (A) and adhesion (B) in vivo in mesenteric veins. Data are mean \pm SEM.
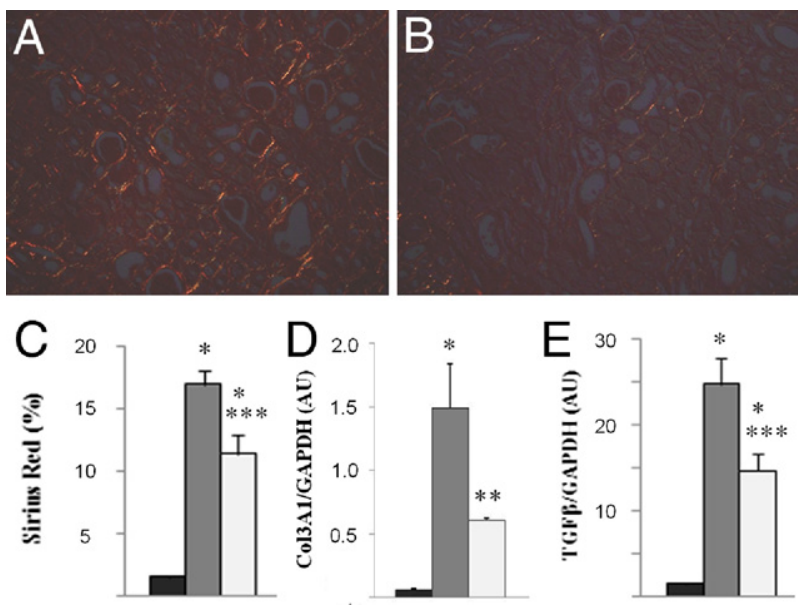

Figure 9. DDR $1^{-/-}$mice are protected against fibrosis induced by UUO Representative views of Sirius red stainings 12 days after UUO in the renal cortex of DDR $1^{-/-}$and WT mice $(\mathbf{A}$ and $\mathbf{B}$, respectively, $\times 20$ ). Morphometric analysis of the percentage of fibrillar collagen in the cortical area (C). Col3A1 (D) and TGF- $\beta 1$ (E) expression evaluated by real-time qPCR. Control nonobstructed kidneys (black bars), WT UUO (gray bars), and DDR1-KO UUO (white bars). Data are mean \pm SEM. ${ }^{*} P<0.01$ versus nonobstructed kidney; ${ }^{* *} P<0.05,{ }^{* * * * *} P<0.01$ versus obstructed WT kidney.

protective effect of DDR1 deletion secondary to its antiinflammatory effect has been also demonstrated in a model of atherosclerosis in mice. ${ }^{8}$ Moreover, Gross et $\mathrm{al}^{25}$ recently studied the role of DDR1 in the kidney of Col4A3-deficient mice and suggested that podocyte-matrix interactions via DDR1 may play a role in glomerular injury in Alport syndrome. DDR1 deficiency was also associated with reduced inflammation in the latter study. Owing to the pathophysiology of the experimental models and to the localization of DDR1 in the kidney, resident vascular smooth muscle cells and glomerular cells expressing DDR1 were respectively susceptible to be the major contributors to the profibrotic action attributed to DDR1 in these studies. Because it is the setting of cellular infiltration and ECM deposition, the interstitium is known to be a major player in the progression of renal fibrosis, irrespective of the initial injury. ${ }^{26}$ To date, the role of DDR1 has not been analyzed in renal diseases resulting from a primitive tubulointerstitial lesion or in models characterized by important interstitial inflammation. In the present work, our aim was to extend the understanding of DDR1 implications in renal injury by specifically analyzing the consequences of the absence of DDR1 in an acute inflammatory tubulointerstitial injury induced by ureteral ligation.

The results of our study show that DDR1 is strongly up-regulated after UUO at both the mRNA and the protein levels. Unexpectedly, we found that the injured tubular epithelial cells do not significantly express DDR1 after UUO. Indeed, the major cells responsible for the de novo induction of DDR1 expression in the kidney are interstitial cells, in particular $\mathrm{F} 4 / 80^{+}$cells. Repeated colocalization studies showed that DDR1 ${ }^{+}$interstitial cells are predominantly macrophages and to a lesser extent dendritic cells. It is well established that the natural history of UUO is characterized by the early onset of renal interstitial inflammation as a consequence of the production of proinflammatory cytokines, such as MCP-1, by resident in- 
terstitial and epithelial cells. ${ }^{27,28}$ Specifically, infiltration by activated macrophages plays a central role in the inflammatory response to UUO and in the progression of the subsequent renal disease. ${ }^{29-32}$ When compared with the renal localization of DDR1 expression in murine hypertension-induced renal disease and in Col4A3-deficient mice, our present results, consistent with recent observations of DDR $1^{+}$macrophages in atherosclerosis, suggest that DDR1 appears to be induced in cell types that are predominantly activated by the specific disease process and involved in the response to the local injury. ${ }^{11,25,33}$

Because we identified an important expression of DDR1 on interstitial macrophages, we hypothesized that the absence of DDR1 in macrophages may protect against UUO-induced lesions in DDR1-deficient mice. To gain insight on the role of DDR1 in macrophages, we performed a step-by-step analysis of the critical phases implicated in macrophage recruitment. We initially demonstrated that DDR1-deficient mice and WT counterparts present similar leukocyte rolling and adhesion in vivo after MCP-1 injection in the absence of differential CCR2 expression on macrophages. We then performed aseptic peritonitis to promote macrophage recruitment, which was significantly blunted in DDR1-deficient mice, similar to the observation of renal macrophage invasion after UUO. We subsequently compared in vitro migration in control macrophages and in macrophages lacking DDR1. In a previous study, macrophages isolated from DDR1-deficient mice presented a reduced invasion index across collagen IV-coated Boyden chambers. ${ }^{33}$ Here, we found that, even in the absence of in vitro activation of DDR1 signaling, macrophages freshly isolated from DDR1 ${ }^{-1-}$ mice exhibited a sustained deficiency in their migratory capacity compared with control macrophages, which implies persistent activation of cellular processes downstream of DDR1. These results show that DDR1 activation in macrophages is required for normal migration and suggest an important role of DDR1 in interstitial macrophage infiltration in inflammatory kidney lesions. An important result of our study was that DDR $1^{-1-}$ and WT macrophages did not exhibit significant differences in M1/M2 polarization. Interestingly IFN- $\gamma$, a cytokine implicated in macrophage cytotoxic activity, presented a strongly reduced expression in the obstructed kidney in DDR1-deficient mice. IFN- $\gamma$ is produced by various cell types implicated in the renal response to UUO, including lymphocytes. In T cells, IFN- $\gamma$ expression is dependent on p38 MAPK, a key pathway activated by DDR1.20,27,34 Alternatively to a role of DDR1 in macrophage migration, but not exclusively, the proinflammatory chemotactic environment may consequently be influenced by DDR1 expression in resident and/or infiltrating cells in UUO.

In our study, the accumulation of fibrillar collagen in the interstitium and the renal expression of type III collagen and TGF- $\beta$, a key profibrotic cytokine, were significantly blunted in DDR1-deficient mice. Sustained interstitial inflammation is associated with the progression of renal fibrosis so that inhibition of inflammation in DDR1-deficient mice may provide an efficient antifibrotic mechanism. ${ }^{26}$ However, alternative factors, including DDR1 expression in resident cells, may also be directly involved in
ECM deposition in this setting. Recent in vivo studies on smooth muscle cells ${ }^{8,35}$ and in vitro ${ }^{7,24}$ results have demonstrated that DDR1 activation resulted in a decrease of matrix accumulation and fibrillogenesis. The effect of DDR1 on matrix production may depend on the cell type and the environment. Owing to the pathophysiology of UUO and to the striking differences in inflammation demonstrated in this study, we believe that our results support the hypothesis that reduced fibrosis in DDR1-deficient mice in this model is predominantly related to reduced renal inflammation.

Together with previous studies that evaluated the role of DDR1 in kidney disease, our results provide evidence that DDR1 is broadly implicated in the pathogenesis of renal fibrosis, irrespective of the compartment of the initial lesion. ${ }^{11,25}$ In perspective, our study underlines a major role of DDR1 in kidney disease progression, particularly through the enhancement of renal inflammation. These results draw attention to the important therapeutic potential of drugs targeting DDR1 in the initiation and the progression of fibrogenesis and probably most importantly in the setting of renal inflammatory diseases.

\section{Acknowledgments}

Transgenic mice were a legacy of Dr. Wolfgang F. Vogel.

\section{References}

1. Vogel WF, Abdulhussein R, Ford CE: Sensing extracellular matrix: an update on discoidin domain receptor function. Cell Signal 2006, 18:1108-1116

2. Lee R, Eidman KE, Kren SM, Hostetter TH, Segal Y: Localization of discoidin domain receptors in rat kidney. Nephron Exp Nephrol 2004 , 97:e62-70

3. Curat CA, Vogel WF: Discoidin domain receptor 1 controls growth and adhesion of mesangial cells. J Am Soc Nephrol 2002, 13:2648-2656

4. Vogel W, Gish GD, Alves F, Pawson T: The discoidin domain receptor tyrosine kinases are activated by collagen. Mol Cell 1997, 1:13-23

5. Hou G, Vogel WF, Bendeck MP: Tyrosine kinase activity of discoidin domain receptor 1 is necessary for smooth muscle cell migration and matrix metalloproteinase expression. Circ Res 2002, 90:1147-1149

6. Agarwal G, Mihai C, Iscru DF: Interaction of discoidin domain receptor 1 with collagen type 1. J Mol Biol 2007, 367:443-455

7. Jonsson M, Andersson T: Repression of Wnt-5a impairs DDR1 phosphorylation and modifies adhesion and migration of mammary cells. J Cell Sci 2001, 114:2043-2053

8. Franco C, Hou G, Ahmad PJ, Fu EYK, Koh L, Vogel WF, Bendeck MP Discoidin domain receptor 1 deletion decreases atherosclerosis by accelerating cell matrix accumulation and reducing inflammation in low-density lipoprotein receptor-deficient mice. During Circ Res 2008, 102:1202-1211

9. Das S, Ongusaha PP, Yang YS, Park JM, Aaronson SA, Lee SW: Discoidin domain receptor 1 receptor tyrosine kinase induces cyclooxygenase-2 and promotes chemoresistance through nuclear factorkappaB pathway activation. Cancer Res 2006, 66:8123-8130

10. Kamohara H, Yamashiro S, Galligan C, Yoshimura T: Discoidin domain receptor 1 isoform-a (DDR1alpha) promotes migration of leukocytes in three-dimensional collagen lattices. FASEB J 2001, 15:2724-2726

11. Flamant M, Placier S, Rodenas A, Curat CA, Vogel WF, Chatziantoniou C, Dussaule JC: Discoidin domain receptor 1 null mice are protected against hypertension-induced renal disease. J Am Soc Nephrol 2006, 17:3374-3381

12. Avivi-Green C, Singal M, Vogel WF: Discoidin domain receptor 1-deficient mice are resistant to bleomycin-induced lung fibrosis. Am J Respir Crit Care Med 2006, 174:420-427 
13. Hou G, Vogel W, Bendeck MP: The discoidin domain receptor tyrosine kinase DDR1 in arterial wound repair. J Clin Invest 2001, 107:727-735

14. Lu KK, Trcka D, Bendeck MP: Collagen stimulates discoidin domain receptor 1-mediated migration of smooth muscle cells through Src. Cardiovasc Pathol 2010, 20:71-76

15. Ahmad PJ, Trcka D, Xue S, Franco C, Speer MY, Giachelli CM, Bendeck MP: Discoidin domain receptor-1 deficiency attenuates atherosclerotic calcification and smooth muscle cell-mediated mineralization. Am J Pathol 2009, 175:2686-2696

16. Vogel W, Aszódi A, Alves F, Pawson T: Discoidin domain receptor 1 tyrosine kinase has an essential role in mammary gland development. Mol Cell Biol 2001, 21:2906-2917

17. Boffa J, Tharaux P, Placier S, Ardaillou R, Dussaule J, Chatziantoniou $\mathrm{C}$ : Angiotensin II activates collagen type I gene in the renal vasculature of transgenic mice during inhibition of nitric oxide synthesis: evidence for an endothelin-mediated mechanism. Circulation 1999 100:1901-1908

18. Chatziantoniou C, Boffa J, Ardaillou R, Dussaule J: Nitric oxide inhibition induces early activation of type I collagen gene in renal resistance vessels and glomeruli in transgenic mice: role of endothelin, J Clin Invest 1998, 101:2780-2789

19. Gross O, Beirowski B, Harvey SJ, McFadden C, Chen D, Tam S, Thorner PS, Smyth N, Addicks K, Bloch W, Ninomiya Y, Sado Y, Weber M, Vogel WF: DDR1-deficient mice show localized subepithelial GBM thickening with focal loss of slit diaphragms and proteinuria. Kidney Int 2004, 66:102-111

20. Matsuyama W, Wang L, Farrar WL, Faure M, Yoshimura T: Activation of discoidin domain receptor 1 isoform $b$ with collagen up-regulates chemokine production in human macrophages: role of p38 mitogenactivated protein kinase and NF-kappa B. J Immunol 2004, 172 2332-2340

21. Hansen C, Greengard P, Nairn A, Andersson T, Vogel W: Phosphorylation of DARPP-32 regulates breast cancer cell migration downstream of the receptor tyrosine kinase DDR1. Exp Cell Res 2006, 312:4011-4018

22. Heinzelmann-Schwarz V, Gardiner-Garden M, Henshall S, Scurry J, Scolyer R, Davies M, Heinzelmann M, Kalish L, Bali A, Kench J, Edwards L, Vanden Bergh P, Hacker N, Sutherland R, O'Brien P: Overexpression of the cell adhesion molecules DDR1, Claudin 3, and Ep-CAM in metaplastic ovarian epithelium and ovarian cancer. Clin Cancer Res 2004, 10:4427-4436

23. Yoshimura T, Matsuyama W, Kamohara H: Discoidin domain receptor 1: a new class of receptor regulating leukocyte-collagen interaction Immunol Res 2005, 31:219-230
24. Flynn L, Blissett A, Calomeni E, Agarwal G: Inhibition of collagen fibrillogenesis by cells expressing soluble extracellular domains of DDR1 and DDR2. J Mol Biol 2010, 395:533-543

25. Gross O, Girgert R, Beirowki B, Kretzler M, Kang HG, Kruegel J, Miosge N, Busse AC, Segerer S, Vogel WF, Muller GA, Weber M: Loss of collagen-receptor DDR1 delays renal fibrosis in hereditary type IV collagen disease. Matrix Biol 2010, 29:346-56

26. Chatziantoniou C, Dussaule JC: Insights into the mechanisms of renal fibrosis: is it possible to achieve regression? Am J Physiol Renal Physiol 2005, 289:F227-E234

27. Chevalier R: Specific molecular targeting of renal injury in obstructive nephropathy. Kidney Int 2006, 70:1200-1201

28. Chevalier R, Forbes M, Thornhill B: Ureteral obstruction as a model of renal interstitial fibrosis and obstructive nephropathy. Kidney Int 2009, 75:1145-1152

29. Vielhauer V, Anders H, Mack M, Cihak J, Strutz F, Stangassinger M, Luckow B, Gröne H, Schlöndorff D: Obstructive nephropathy in the mouse: progressive fibrosis correlates with tubulointerstitial chemokine expression and accumulation of CC chemokine receptor 2- and 5-positive leukocytes. J Am Soc Nephrol 2001, 12:1173-1187

30. Lange-Sperandio B, Cachat F, Thornhill B, Chevalier R: Selectins mediate macrophage infiltration in obstructive nephropathy in newborn mice. Kidney Int 2002, 61:516-524

31. Lange-Sperandio B, Schimpgen K, Rodenbeck B, Chavakis T, Bierhaus A, Nawroth $P$, Thornhill B, Schaefer F, Chevalier R: Distinct roles of Mac-1 and its counter-receptors in neonatal obstructive nephropathy. Kidney Int 2006, 69:81-88

32. Anders $H$, Vielhauer $V$, Frink $M$, Linde $Y$, Cohen $C$, Blattner $S$, Kretzler M, Strutz F, Mack M, Gröne H, Onuffer J, Horuk R, Nelson $\mathrm{P}$, Schlöndorff D: A chemokine receptor CCR-1 antagonist reduces renal fibrosis after unilateral ureter ligation. J Clin Invest 2002 109:251-259

33. Franco C, Britto K, Wong E, Hou G, Zhu SN, Chen M, Cybulsky MI, Bendeck MP: Discoidin domain receptor 1 on bone marrow-derived cells promotes macrophage accumulation during atherogenesis. Circ Res 2009, 105:1141-1148

34. Rincón M, Enslen H, Raingeaud J, Recht M, Zapton T, Su M, Penix L, Davis R, Flavell R: Interferon-gamma expression by Th1 effector T cells mediated by the p38 MAP kinase signaling pathway. EMBO $1998,17: 2817-2829$

35. Franco C, Ahmad PJ, Hou G, Wong E, Bendeck MP: Increased cell and matrix accumulation during atherogenesis in mice with vessel wall-specific deletion of discoidin domain receptor 1. Circ Res 2010 106:1775-1783 